\title{
CANONICAL EMBEDDINGS OF PAIRS OF ARCS
}

\author{
MARIO BONK AND ALEXANDRE EREMENKO \\ Dedicated to the memory of Walter Hayman
}

\begin{abstract}
We show that for given four points in the Riemann sphere and a given isotopy class of two disjoint arcs connecting these points in two pairs, there exists a unique configuration with the property that each arc is a hyperbolic geodesic segment in the complement of the other arc.
\end{abstract}

In the recent paper PW20, Peltola and Wang made a remarkable restatement of results in [EG02, EG11 about the existence and uniqueness of a real rational function with prescribed real critical points. To formulate this precisely, we consider chord diagrams in the closure $\overline{\mathbb{D}}$ of the unit disk $\mathbb{D}=\{z \in \mathbb{C}:|z|<1\}$ in the complex plane $\mathbb{C}$. Such a chord diagram has prescribed points $a_{1}, \ldots, a_{2 d-2}, d \geqslant 2$, on the unit circle and $d-1$ disjoint crosscuts $e_{1}, \ldots, e_{d-1}$ in $\overline{\mathbb{D}}$ connecting pairs of these points. We call such a chord diagram canonical if every crosscut $e_{k}$ is a hyperbolic geodesic in the unique component of $\mathbb{D} \backslash \bigcup_{j \neq k} e_{j}$ that contains the interior points of $e_{k}$.

Theorem 1.1 in [PW20] states that for any prescribed points $a_{1}, \ldots, a_{2 d-2}$ there is a unique canonical chord diagram in every combinatorial class. This canonical chord diagram can be obtained from the preimage of the real line under a real rational function of degree $d$ with critical points at $a_{1}, \ldots, a_{2 d-2}$. This theorem has important applications for the study of the Stochastic Loewner Evolution (SLE).

The number of combinatorial classes of chord diagrams with prescribed vertices is finite: it is the Catalan number. In this note we give a simple example of a similar problem with four prescribed points and infinitely many canonical configurations.

Our configurations consist of four distinct points $a_{0}, a_{1}, a_{2}, a_{3}$ in the Riemann sphere $\widehat{\mathbb{C}}=\mathbb{C} \cup\{\infty\}$ and two disjoint arcs $\gamma_{0}$ and $\gamma_{1}$, where $\gamma_{0}$ has the endpoints $a_{0}$ and $a_{1}$, and $\gamma_{1}$ has the endpoints $a_{2}$ and $a_{3}$. We say that two such configurations are equivalent if the points are the same, and the arcs of

Date: September 6, 2021.

2010 Mathematics Subject Classification. Primary: 30F45, 60J67, 81T40.

Key words and phrases. Conformal map, hyperbolic metric, conic singularity.

M.B. was partially supported by NSF grants DMS-1808856 and DMS-2054987. 
the first configuration can be deformed into the arcs of the second configuration by an isotopy of the sphere that keeps the endpoints of the arcs fixed. A configuration is called canonical if for each $k \in\{0,1\}$ the arc $\gamma_{k}$ is a hyperbolic geodesic segment in the simply connected hyperbolic region $\widehat{\mathbb{C}} \backslash \gamma_{1-k}$.

Theorem. For every equivalence class of configurations, there exists a unique canonical configuration.

Our proof will show that one can obtain an explicit description of canonical configurations as follows. We may assume without loss of generality that

$$
\left(a_{0}, a_{1}, a_{2}, a_{3}\right)=\left(\infty, e_{1}, e_{2}, e_{3}\right),
$$

where $e_{1}, e_{2}, e_{3} \in \mathbb{C}$ and $e_{1}+e_{2}+e_{3}=0$. Let $\wp$ be the Weierstrass function satisfying

$$
\left(\wp^{\prime}\right)^{2}=4\left(\wp-e_{1}\right)\left(\wp-e_{2}\right)\left(\wp-e_{3}\right) .
$$

We denote the line segment joining two points $z, w \in \mathbb{C}$ by $[z, w]$. Then each canonical configuration for the points as in (1) has the form

$$
\gamma_{0}=\wp\left(\left[0, \omega_{1} / 2\right]\right), \quad \gamma_{1}=\wp\left(\left[\omega_{2} / 2,\left(\omega_{1}+\omega_{2}\right) / 2\right]\right),
$$

where the pair $\omega_{1}$ and $\omega_{2}$ generates the period lattice of $\wp$, and $e_{1}=\wp\left(\omega_{1} / 2\right)$, $e_{2}=\wp\left(\omega_{2} / 2\right)$, and $e_{3}=\wp\left(\left(\omega_{1}+\omega_{2}\right) / 2\right)$.

In order to prove our theorem, we first state some auxiliary facts. An anti-conformal involution of a region $D$ in the Riemann sphere $\widehat{\mathbb{C}}$ is an anticonformal homeomorphism $\sigma$ of $D$ onto itself such that $\sigma \circ \sigma=\operatorname{id}_{D}$, where $\operatorname{id}_{D}$ denotes the identity map on $D$.

We will use the following well-known facts about anti-conformal involutions.

Lemma 1. Let $D$ be a simply connected hyperbolic region in $\widehat{\mathbb{C}}$, and $\sigma$ be an anti-conformal involution of $D$. Then the set of fixed points of $\sigma$ is a hyperbolic geodesic. Conversely, for every hyperbolic geodesic there exists a unique anticonformal involution of $D$ that fixes all points on this geodesic.

Proof. By the Riemann mapping theorem, we may assume that $D$ is the unit disk $\mathbb{D}$. If $C$ is a hyperbolic geodesic in $\mathbb{D}$, then $C$ is an arc of a circle or a line segment that is orthogonal to the unit circle $\partial \mathbb{D}$. Then reflection in $C$ is an anti-conformal involution $\sigma_{C}$ of $\mathbb{D}$ that fixes every point of $C$.

Note that if $C=I=(-1,1)$, then $\sigma_{I}(z)=\bar{z}$ and so the anti-conformal involution $\sigma_{I}$ is a hyperbolic isometry.

Now let $\sigma$ be an arbitrary anti-conformal involution of $\mathbb{D}$. Then $\sigma$ is also a hyperbolic isometry. Indeed, $\tau=\sigma \circ \sigma_{I}$ is a conformal automorphism of $\mathbb{D}$ and hence a hyperbolic isometry. This implies that $\sigma=\tau \circ \sigma_{I}$ is a hyperbolic isometry as well. 
This in turn implies that $\sigma$ has a fixed point $w_{0} \in \mathbb{D}$, namely, for $w_{0}$ we can take the midpoint of the hyperbolic geodesic segment $S$ joining some point $z_{0} \in \mathbb{D}$ with $\sigma\left(z_{0}\right) \in \mathbb{D}$. To see this, note that $\sigma$ is an isometry on $S=\sigma(S)$ that interchanges the endpoints of $S$.

By conjugating with an auxiliary automorphism, we may assume that $w_{0}=$ 0 . Then $\tau=\sigma \circ \sigma_{I}$ is an automorphism of $\mathbb{D}$ that fixes 0 . Hence $\tau(z)=e^{i \theta} z$ with $\theta \in \mathbb{R}$. It follows that $\sigma(z)=\left(\tau \circ \sigma_{I}\right)(z)=e^{i \theta} \bar{z}$, and so $\sigma$ is equal to the reflection $\sigma_{C}$ in the hyperbolic geodesic $C=\left\{e^{i \theta / 2} t:-1<t<1\right\}$. In particular, $\sigma$ fixes the points in $C$ and no other points.

The argument also shows that each anti-conformal involution of $\sigma$ of $\mathbb{D}$ has the form $\sigma=\sigma_{C}$ for some hyperbolic geodesic $C$. This implies that the fixed point set of $\sigma$ uniquely determines $\sigma$.

Lemma 2. An anti-conformal involution $\sigma$ of an annulus $A=\{z \in \mathbb{C}: 1<$ $|z|<R\}$ with $R>1$ that leaves each boundary component invariant is of the form $\sigma(z)=e^{i \theta} \bar{z}$ with $\theta \in \mathbb{R}$.

Note that a priori the involution $\sigma$ is not defined on the boundary of $A$; so by invariance of the boundary components we mean that $\sigma(z) \rightarrow \partial_{k} A$ as $z \in A \rightarrow \partial_{k} A$ for each boundary component $\partial_{k} A=\left\{z \in \mathbb{C}:|z|=R^{k}\right\}$ of $A$ for $k=0,1$.

Proof. Let $\tau(z)=\bar{z}$. Then $\rho=\sigma \circ \tau$ is a conformal automorphism of $A$ that preserves the boundary components of $A$. It is well-known that then $\rho(z)=e^{i \theta} z$ with $\theta \in \mathbb{R}$. Hence $\sigma(z)=(\rho \circ \tau)(z)=e^{i \theta} \bar{z}$.

Proof of the Theorem. We assume that we have some canonical configuration. We will analyze the situation and will obtain an explicit description from which existence and uniqueness will be evident.

So suppose the disjoint arcs $\gamma_{1}$ and $\gamma_{2}$ in $\widehat{\mathbb{C}}$ form a canonical configuration. Then by Lemma 1, there exists an anti-conformal involution $\sigma_{k}: \widehat{\mathbb{C}} \backslash \gamma_{1-k} \rightarrow$ $\widehat{\mathbb{C}} \backslash \gamma_{1-k}$ fixing the points in $\gamma_{k}$ for $k \in\{0,1\}$.

If we restrict these maps to the ring domain $D=\widehat{\mathbb{C}} \backslash\left(\gamma_{0} \cup \gamma_{1}\right)$, then we obtain anti-conformal involutions of $D$ fixing the boundary components. Now $D$ is conformally equivalent to an annulus $A=\{z: 1<|z|<R\}$ with $R>1$. Then by Lemma 2, each anti-conformal involution $\sigma_{k}$ on $D$ corresponds to a reflection $\tau_{k}$ in a line through the origin on $A$.

Conversely, suppose that $\tau_{0}$ and $\tau_{1}$ are two reflections in lines through the origin. Then we can identify or "weld" the points on each boundary component $\partial_{k} A$ of $A$ together by using the map $\tau_{k}$ for $k=0,1$. The quotient space carries a natural conformal structure, and is hence conformally equivalent to the Riemann sphere by the uniformization theorem. This sphere will carry two distinguished arcs $\gamma_{k}, k=0,1$, corresponding to each boundary $\partial_{k} A$ after 
the welding. Note that each reflection $\tau_{k}$ passes to the quotient of $A \cup \partial_{k} A$ as an anti-conformal involution fixing the points corresponding to points on $\partial_{k} A$. This induces an anti-conformal involution of $\widehat{\mathbb{C}} \backslash \gamma_{1-k}$ fixing the points on $\gamma_{k}$. By Lemma 1, the arc $\gamma_{k}$ is a hyperbolic geodesic segment in the hyperbolic region $\widehat{\mathbb{C}} \backslash \gamma_{1-k}$. It follows that $\gamma_{0}$ and $\gamma_{1}$ form a canonical configuration.

We have shown that the canonical configurations are precisely those that can be obtained from an annulus $A=\{z \in \mathbb{C}: 1<|z|<R\}, R>1$, by welding the points in each boundary component $\partial_{k} A$ together by using a reflection $\tau_{k}$ in a line through the origin for $k=0,1$.

Now a (closed) annulus $\bar{A}=\{z \in \mathbb{C}: 1 \leqslant|z| \leqslant R\}$ carries an essentially unique (up to scaling) flat conformal metric in which the circles $\{z \in \mathbb{C}:|z|=$ $\left.R^{k}\right\}, k=0,1$, are geodesics. It has length element

$$
|d z| /|z| \text {. }
$$

The annulus $\bar{A}$ equipped with this metric is isometric to the cylinder

$$
\left\{\left(x_{1}, x_{2}, x_{3}\right) \in \mathbb{R}^{3}: x_{1}^{2}+x_{2}^{2}=1,0 \leqslant x_{3} \leqslant \log R\right\}
$$

with the Riemannian metric induced from $\mathbb{R}^{3}$.

For the proof of the essential uniqueness of such a metric on $\bar{A}$, one extends it to $\mathbb{C}^{*}=\mathbb{C} \backslash\{0\}$ by successive reflections and then lifts it to the universal cover $\mathbb{C}$ by the exponential map. The resulting conformal metric on $\mathbb{C}$ will be complete and flat, and hence equal to the Euclidean metric up to a scaling (see Huber [Hu66] for an analytic approach). A representation of the conformal metric on $\bar{A}$ as in (4) follows.

After the welding of the boundary components $\partial_{k} A$ by each reflection $\tau_{k}$, the flat metric in (4) descends to a flat metric on the quotient space with possible singularities in the points of $\gamma_{0} \cup \gamma_{1}$. Since $\partial_{k} A$ is a geodesic in the flat metric (4), $\gamma_{k}$ will be a geodesic arc in this metric with conic singularities at the endpoints and the angles at these singularities are $\pi$.

So we obtain the following geometric description of canonical configurations: to each canonical configuration corresponds a flat metric on the sphere with four conic singularities at $a_{0}, a_{1}, a_{2}, a_{3}$ with cone angle $\pi$ such that $\gamma_{0}$ and $\gamma_{1}$ are geodesic segments.

The converse is also true: given a flat metric on the sphere with four conic singularities $a_{0}, a_{1}, a_{2}, a_{3}$ with cone angles $\pi$, any pair of disjoint geodesics $\gamma_{0}$ connecting $a_{0}$ with $a_{1}$, and $\gamma_{1}$ connecting $a_{2}$ with $a_{3}$ is a canonical configuration.

To see this, note that we can cut open the sphere along the $\operatorname{arcs} \gamma_{0}$ and $\gamma_{1}$. Then up to scaling, $D=\widehat{\mathbb{C}} \backslash\left(\gamma_{0} \cup \gamma_{1}\right)$ equipped with the flat metric is isometric to an annulus $A=\{z \in \mathbb{C}: 1<|z|<R\}$ with $R>1$, equipped with the metric (4). Here each geodesic $\gamma_{k}$ is doubled and represented by two 
circular arcs $\alpha_{k}$ and $\alpha_{k}^{\prime}$ of equal length that have common endpoints and whose union is a boundary component of $A$. We may assume that $\gamma_{k}$ corresponds to $\partial_{k} A=\alpha_{k} \cup \alpha_{k}^{\prime}$ for $k=0,1$. The sphere $\widehat{\mathbb{C}}$ with the flat metric and the geodesic arcs $\gamma_{0}$ and $\gamma_{1}$ can be recovered from $A$ if we identify correspond points on $\alpha_{k}$ and $\alpha_{k}^{\prime}$ by an isometry fixing the common endpoints of $\alpha_{k}$ and $\alpha_{k}^{\prime}$. But such an isometry is necessarily given by a reflection $\tau_{k}$ in a line though the origin. So we are back to our first description of canonical configurations as a quotient space of an annulus $A$.

A flat metric on the sphere $\widehat{\mathbb{C}}$ with four prescribed conic singularities with angles $\pi$ gives $\widehat{\mathbb{C}}$ the structure of a parabolic orbifold. The corresponding flat metric is unique up to scaling, and obtained by pushing the Euclidean metric in the plane forward by the universal orbifold covering map $\Theta: \mathbb{C} \rightarrow$ $\widehat{\mathbb{C}}$. For a parabolic orbifold with four conic singularities with angles $\pi$ this universal orbifold covering map $\Theta$ is a Weierstrass $\wp$-function followed by a Möbius transformation. With the normalization (11), we actually have $\Theta=\wp$, where $\wp$ is as in (2). Then the length element of the flat metric is given by $d s=\left|\wp^{\prime}(z)\right||d z|$ and geodesic segments on $\widehat{\mathbb{C}}$ in the flat metric are given by images of Euclidean geodesic segments under $\wp$ (for a thorough discussion of the relevant facts about orbifolds see [BM17, Sections 3.5, A.9, A.10]).

For the given normalization (1), the arc $\gamma_{0}$ in a canonical configuration lifts under $\wp$ to a Euclidean line segment $\left[z_{0}, z_{1}\right] \subseteq \mathbb{C}$ such that $\wp$ is an isometry of $\left[z_{0}, z_{1}\right]$ onto $\gamma_{0}$. Here we may assume that $\wp\left(z_{0}\right)=\infty$ and $\wp\left(z_{1}\right)=e_{1}$.

Let $\Gamma \subseteq \mathbb{C}$ be the period lattice of $\wp$. Since we have translation invariance of $\wp$ under $\Gamma$ and $\wp^{-1}(\infty)=\Gamma$, we may further assume that $z_{0}=0$. Since $\wp\left(z_{1}\right)=e_{1}$, the point $z_{1}$ must be a half-period of $\wp$, i.e., $z_{1} \in \frac{1}{2} \Gamma$. Now $\wp$ is injective on $\left[z_{0}, z_{1}\right]=\left[0, z_{1}\right]$, and so the point $z_{1}$ must be of the form $z_{1}=\omega_{1} / 2$, where $\omega_{1} \neq 0$ is a primitive element of $\Gamma$, i.e., $\omega_{1}$ cannot be represented in the form $\omega_{1}=n \gamma$ with $n \in \mathbb{N}, n \geqslant 2$, and $\gamma \in \Gamma$. It follows that $\gamma_{0}=\wp\left(\left[0, \omega_{1} / 2\right)\right]$ as in (3).

Since $\omega_{1}$ is a primitive element of $\Gamma$, there exists an element $\omega_{2} \in \Gamma$ such that $\omega_{1}$ and $\omega_{2}$ form a basis of $\Gamma$. For given $\omega_{1}$, the choice of $\omega_{2}$ is not unique, but if we make one choice for $\omega_{2}$, then all other choices $\omega_{2}^{\prime}$ are of the form

$$
\omega_{2}^{\prime}= \pm \omega_{2}+n \omega_{1}
$$

with $n \in \mathbb{Z}$. Note that $\wp$ maps the half-periods $\frac{1}{2} \omega_{2}^{\prime}$ to $e_{2}$ or $e_{3}$. With suitable choice of $\omega_{2}$ we may assume that $\wp\left(\omega_{2} / 2\right)=e_{2}$.

We now lift the second arc $\gamma_{1}$ in our canonical configuration under $\wp$ to a line segment $\left[z_{2}, z_{3}\right] \subseteq \mathbb{C}$ starting at $z_{2}=\omega_{2} / 2$. Here $\wp\left(z_{3}\right)=e_{3}$, and so $z_{3} \in \frac{1}{2} \Gamma$ is a half-period.

We can say more here. Since the $\wp$-function satisfies

$$
\wp( \pm z+\alpha)=\wp(z) \text { for } z \in \mathbb{C} \text { and } \alpha \in \Gamma,
$$


it is invariant under reflections in half-periods. This implies that $\wp^{-1}\left(\gamma_{0}\right)$ contains the full line passing through $z_{0}=0$ and $z_{1}=\omega_{1} / 2$. Similarly, $\wp^{-1}\left(\gamma_{1}\right)$ contains the full line passing through $z_{2}=\omega_{2} / 2$ and the half-period $z_{3}$. Since $\gamma_{0}$ and $\gamma_{1}$ are disjoint, these lines cannot meet and hence must be parallel. It follows that $z_{3}$ necessarily has the form $z_{3}=\frac{1}{2}\left(n \omega_{1}+\omega_{2}\right)$ with $n \in \mathbb{Z}$. Since $\wp\left(z_{3}\right)=e_{3}$, the integer $n$ must be odd, and since $\wp$ is injective on the lift $\left[z_{2}, z_{3}\right]$, we must have $z_{3}=\frac{1}{2}\left( \pm \omega_{1}+\omega_{2}\right)$. By reflection symmetry of $\wp$ in the half-period $z_{2}=\omega_{2} / 2$ and replacing the original lift $\left[z_{2}, z_{3}\right]$ by its reflection image in $z_{2}$, we may assume that $z_{3}=\frac{1}{2}\left(\omega_{1}+\omega_{2}\right)$.

We conclude that under the normalization (11), arcs in a canonical configuration $\gamma_{0}$ and $\gamma_{1}$ have the form (3). Conversely, arcs as in (3) form a canonical configuration as follows from our geometric description of canonical configuration in terms of flat metrics on $\widehat{\mathbb{C}}$.

It remains to show that under the assumption (11) we obtain exactly one canonical configuration of arcs in each isotopy class.

Our previous analysis shows that in (3) the arcs $\gamma_{0}$ and $\gamma_{1}$ are uniquely determined once we know the primitive element $\omega_{1}$ up to sign. Indeed, it is clear that this determines $\gamma_{0}$. Moreover, the choice of $\omega_{1}$ up to sign does not determine $\omega_{2}$ uniquely, but it follows from (5) and (6) that the arc $\gamma_{2}=$ $\left[\wp\left(\omega_{2} / 2,\left(\omega_{1}+\omega_{2}\right) / 2\right]\right.$ is uniquely determined independent of the choice of the sign of $\omega_{1}$ and the choice of $\omega_{2}$.

Now suppose we have chosen a fixed basis $\omega_{1}^{0}$ and $\omega_{2}^{0}$ of the period lattice $\Gamma$. Then the primitive elements $\omega_{1}$ of $\Gamma$ are precisely the elements of the form

$$
\omega_{1}=r \omega_{1}^{0}+s \omega_{2}^{0}
$$

where $r, s \in \mathbb{Z}$ are relatively prime. By choosing the appropriate sign of $\omega_{1}$, we may assume that $s \geqslant 0$ and that $r=1$ if $s=0$. The ratio $r / s \in \widehat{\mathbb{Q}}=\mathbb{Q} \cup\{\infty\}$ describes the slope of the line in $\mathbb{C}$ passing through 0 and $\omega_{1}$ and we have a bijective correspondence between slopes $r / s \in \widehat{\mathbb{Q}}$ and primitive elements $\pm \omega_{1}$ of $\Gamma$ up to sign.

Now it is a well known fact that isotopy classes of arcs in a sphere wit $\mathrm{h}$ four marked points are in one-to-one correspondence with these rational slopes $r / s \in \widehat{\mathbb{Q}}$ (see [FM12, Chapter 2] for a related discussion). In our case, $\pm \omega_{1} \leftrightarrow \wp\left(\left[0, \omega_{1} / 2\right]\right)$ induces a bijective correspondence between primitive elements $\pm \omega_{1}$ of $\Gamma$ up to sign and isotopy classes of arcs $\gamma_{0}$ in $\widehat{\mathbb{C}}$ with marked points as (11) (see [BHI21, Section 2.6] for a thorough discussion in the spirit of the present considerations). Note that the isotopy class of $\gamma_{0}$ uniquely determines the isotopy class of the pair $\left(\gamma_{0}, \gamma_{1}\right)$. The statement follows.

Acknowledgment. We thank Daniel Meyer for his remarks on this paper. 


\section{REFERENCES}

[BHI21] M. Bonk, M. Hlushchanka, and A. Iseli, Eliminating Thurston obstructions and controlling dynamics on curves, Preprint, arXiv:2006.08574, 2021.

[BM17] M. Bonk and D. Meyer, Expanding Thurston Maps. Mathematical Surveys and Monographs, Vol. 225, Amer. Math. Soc., Providence, RI, 2017.

[EG02] A. Eremenko and A. Gabrielov, Rational functions with real critical points and the B. and M. Shapiro conjecture in real enumerative geometry, Ann. Math. 155 (2002), $105-129$.

[EG11] A. Eremenko and A. Gabrielov, An elementary proof of the B. and M. Shapiro conjecture for rational functions, in: Notions of positivity and the geometry of polynomials, Birkhäuser/Springer, Basel, 2011, pp. 167-178.

[FM12] B. Farb and D. Margalit, A primer on mapping class groups, Princeton Univ. Press, Princeton and Oxford, 2012.

[Hu66] A. Huber, Vollständige konforme Metriken und isolierte Singularitäten subharmonischer Funktionen, Comment. Math. Helv. 41 (1966/67), 105-136.

[PW20] E. Peltola and Y. Wang, Large deviations of multichordal $S L E_{0+}$, real rational functions, and zeta-regularized determinants of Laplacians, Preprint, arXiv:2006.08574, 2020 .

Department of Mathematics, University of California, los Angeles, CA 90095

Email address: mbonk@math.ucla.edu

Department of Mathematics, Purdue University, West Lafayette, IN 47907

Email address: eremenko@purdue.edu 